\title{
Self-reported hearing loss and manual audiometry: a rural versus urban comparison
}

Christopher G. Brennan-Jones BSc(Hons) ${ }^{1,2, *}$, Dunay S. Taljaard AuD, $\mathrm{PhD}^{1,2,4}$,

Sophie E.F. Brennan-Jones MBChB ${ }^{1,2,3}$, Rebecca J. Bennett MAud ${ }^{1,2}$,

De Wet Swanepoel $\mathrm{PhD}^{1,2,5}$ and Robert H. Eikelboom $\mathrm{PhD}^{1,2,5}$

\footnotetext{
${ }^{1}$ Ear Science Institute Australia, Perth, Western Australia, Australia

${ }^{2}$ Ear Sciences Centre, School of Surgery, The University of Western Australia, Perth, Western Australia, Australia

${ }^{3}$ School of Population Health, The University of Western Australia, Perth, Western Australia, Australia

${ }^{4}$ Department of Audiology, Princess Margaret Hospital, Perth, Western Australia, Australia

${ }^{5}$ Department of Speech-Language Pathology and Audiology, University of Pretoria, Pretoria, South Africa
}

*Correspondence: Mr Christopher Brennan-Jones, Ear Science Institute Australia, Ear Sciences Centre, School of Surgery, The University of Western Australia, CRICOS Provider Code: 00126G, Suite 1, Level 2, 1 Salvado Road, Subiaco, Western Australia, 6008, Australia. Email: chris.brennan-jones@earscience.org.au

Declaration of conflict interest: No personal financial interest for any authors. People failing in their hearing screening were advised to seek further medical or audiological advice; the Lions Hearing Clinics, Ear Science Institute Australia, was one of the recommended referral sites.

\section{ABSTRACT:}

Objective: To examine whether self-reported hearing difficulty is an accurate measure of hearing loss compared to standard hearing screening with pure tone audiometry in rural and urban communities.

Design: Convenience sampling.

Setting: Urban and rural areas of Western Australia.

Participants: 2090 participants (923 male; 1165 female; 2 unknown) aged 20 to 100 years presenting for community-based hearing screening in urban (982) and rural (1090) areas. Main outcome measures: Self-reported hearing difficulty assessed with the Hearing Handicap in the Elderly-Screening (HHIE-S) questionnaire. Hearing loss defined as average hearing thresholds $>25 \mathrm{~dB}$ in the better ear using screening audiometry conducted at 500 , 1000, 2000 and $4000 \mathrm{~Hz}$.

Results: The HHIE-S was sensitive $(\geq 60 \mathrm{yrs}=76.69 \%$; $<60 \mathrm{yrs}=71.67 \%)$ but not specific $(\geq 60 \mathrm{yrs}=45.15 \% ;<60 \mathrm{yrs}=49.63 \%)$ for identifying hearing loss. The $<60$ age group had a 
hearing loss prevalence of $25.6 \%$, and a false-positive rate of $67.12 \%$ compared to a prevalence of $69.12 \%$ and false-positive rate of $29.77 \%$ for the $\geq 60$ age group. For all ages, rural participants were more likely to have a disabling hearing loss (OR 2.04 [95\%CI 1.55, 2.67]; $X^{2}(1)=27.28 ; p<0.001$ ) but there were no significant differences in hearing aid uptake. Conclusions: Patients in rural areas presenting for hearing screenings are more likely to suffer a hearing loss than adults in urban areas. We suggest rural health practitioners incorporate a self-reported hearing loss questionnaire into health check-ups for adults, particularly patients aged $\geq 60$ years due to the high prevalence of hearing loss in this group.

\section{What is already known on this subject?}

- Access to treatment for hearing loss may be limited in rural and remote areas

- Intervention for hearing loss in the pre-retirement period is associated with better patient outcomes

- Early identification of hearing loss in primary care using the standard audiometric screening can be costly, requiring specialised equipment and training

\section{What this study adds:}

- There is a significant difference in the presence hearing loss in rural populations compared to urban populations in a self-referring participants

- The prevalence of self-reported hearing loss in adults aged over 60 years is extremely high in a self-referring population

- Self-report measures are a cost-effective method of screening for hearing loss in primary care and identifying patients but lack accuracy compared to audiometric screening

\section{INTRODUCTION}

Approximately 15\% of the world's adult population have some degree of hearing loss, half of which is considered to be disabling. ${ }^{1}$ This makes hearing loss one of the top twenty causes of disease burden globally. ${ }^{1}$ Hearing loss affects interpersonal communication, psychosocial wellbeing, quality of life and economic independence. ${ }^{2}$ The lack of access and availability of appropriate interventions for patients, particularly those in remote and rural areas, is of significant concern. ${ }^{3}$

Screening for hearing loss involves audiological tests that are costly and are often not available in rural healthcare settings. ${ }^{4}$ Other screening methods, such as telephone-based hearing screening and the whispered voice test, have been shown to be a valid method of screening for hearing loss. However, telephone-based screening requires initiation from the patient and the whispered voice test is often dependent on clinician experience. ${ }^{5,6}$ Self-report questionnaires for identifying hearing loss have therefore been proposed as a cost-effective 
and time-efficient method of audiological screening that can be utilised by any health professional group. ${ }^{7-9}$

The uptake of hearing aids by patients with hearing loss is low. ${ }^{10}$ Identification and intervention for hearing loss in the pre-retirement period (approximately 50 to 65 years of age) is associated with a greater uptake of hearing aids ${ }^{11}$ and improved rehabilitation outcomes. $^{2-5}$ Self-report questionnaires may therefore be useful in early identification of hearing loss, prompting early intervention and improved outcomes in those who are motivated to receive treatment and rehabilitation for their hearing loss.

The Hearing Handicap Inventory for the Elderly - Screening (HHIE-S) ${ }^{12}$ is a widely used measure of self-reported hearing difficulty and has been adapted for different age and cultural groups. ${ }^{13}$ It has been extensively psychometrically validated, ${ }^{12}$ has comparable accuracy to similar validated questionnaires ${ }^{14}$ and higher accuracy than a single question for diagnosing hearing impairment. ${ }^{15}$

This study was designed to evaluate the accuracy of self-report measure for determining hearing loss amongst self-referring patients compared to audiometric screening and to examine the association between the uptake of hearing aids and location (rural or urban).

\section{METHODS}

\section{Study Cohort}

Data from hearing screenings conducted on self-referred volunteers performed by the Lions Hearing Foundation in Western Australia between March 2010 and July 2013 were collated in this study. Excluded were 182 potential participants who declined permission for their data to be used, 5 who did not complete the audiometric screening, 11 who did not provide a date of birth, and 49 who were under the age 20 years; leaving a cohort of 2090 participants. For the purposes of this study, participants were divided into two groups at 60 years of age, broadly representing a pre-retirement group.

\section{Materials and procedures}

All participants completed the HHIE-S, a conventional audiometric hearing screening and an additional questionnaire including items on hearing aid use and outcomes designed by the investigators. HHIE-S scores of $>8$ indicated a hearing impairment. Ears were examined by 
otoscopy to ensure there was no obstruction of the ear canal prior to screening audiometry, which was conducted with a GSI 17 audiometer (Grasson Stadler, Eden Prairie, MN, USA) and Peltor 211 headphones (3M, St. Paul, MN, USA), calibrated annually. Screening was conducted by trained, non-specialist volunteers in a sound-treated booth on a bus modified for mobile hearing screenings. Hearing screening commenced at $25 \mathrm{dBHL}$. Participants "passed" if they responded to low intensity sounds at 500,1000, 2000 and $4000 \mathrm{~Hz}$ in both ears. If they did not respond, intensity was increased in $5 \mathrm{dBHL}$ steps until the participant responded. Order of testing left or right ears first was not prescribed, and the order of testing frequencies was 500, 1000, 2000 and $4000 \mathrm{~Hz}$.

\section{Data analysis}

Hearing screening and HHIE-S data, together with age, gender, location and hearing aid use were analysed in SPSS v21 (New York: IBM Corp). Hearing loss was defined as mild (best ear four-frequency average $>25 \mathrm{~dB}$ ) or disabling (best ear four-frequency average $>40 \mathrm{~dB}$ ) across the frequencies of 500, 1000, 2000 and $4000 \mathrm{~Hz}$, according to the WHO criteria. ${ }^{1}$ Accessibility/Remoteness Index of Australia (ARIA) classification codes ${ }^{16}$ were used to identify an urban (ARIA code: 0 ) or rural/regional address (ARIA codes: 1, 2, 3, 4) according to postcode.

\section{RESULTS}

\section{Participant profile}

Of the 2090 participants in the study, 982 (46.99\%) resided in the metropolitan area of Perth and $1090(52.15 \%)$ in the regional, rural or remote areas of Western Australia according to ARIA classifications; $18(0.86 \%)$ people did not provide a valid postcode for ARIA classification. More females $(n=1165)$ than males $(n=923)$ were included; the sex of two people was not reported. The mean age of participants was 60.0 years (SD 13.93, range 20.3 to 100.32 years), with no statistically significant difference between the gender balances or age of urban versus rural participants (independent sample t-tests; $p>0.05$ ). Figure 1 shows the distribution of participants according to age, gender and hearing loss. 


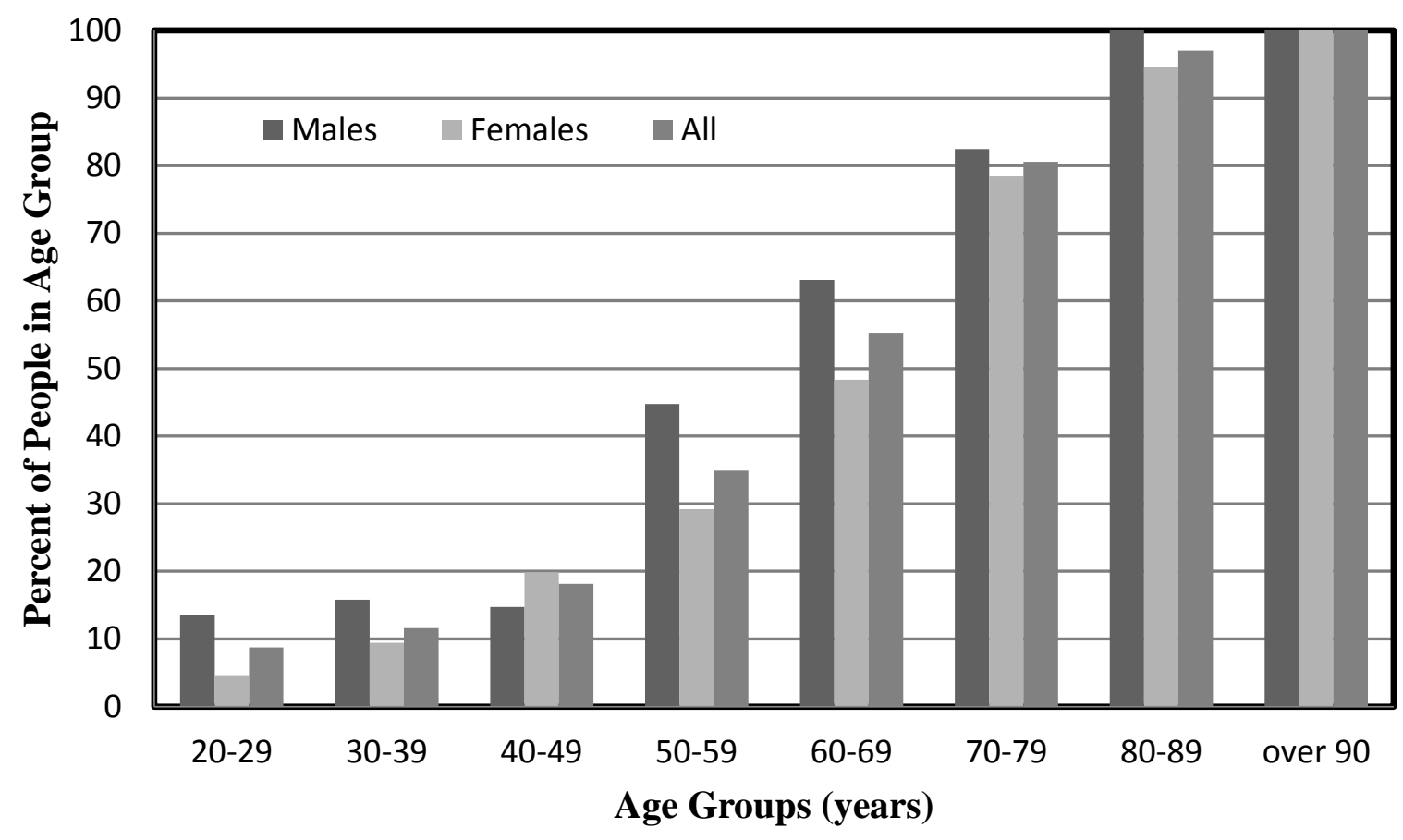

Figure 1: Prevalence of hearing loss (>25dBHL) according to age and sex in participants attending the Lions Hearing Bus.

Accuracy of HHIE-S self-report questionnaire

The sensitivity of the HHIE-S questionnaire was 76.69\% (95\%CI 73.33, 79.75), with specificity of $45.15 \%$ (95\%CI 40.29, 50.01) for identifying mild hearing loss in adults aged $\geq 60$ years. For adults aged $<60$ years, the sensitivity of the HHIE-S was $71.67 \%$ (95\%CI $65.35,77.27)$ and specificity was $49.63 \%$ (95\%CI 45.80, 53.46). The $<60$ group had a falsepositive rate of $67.12 \%$ [95\%CI $62.82,71.16$ ] compared to $29.77 \%$ [95\%CI \% 26.57, 33.19] for the $\geq 60$ group; the false-negative rate for the $<60$ year old group was $16.42 \%$ [95\%CI $13.01,20.48]$ and for the $\geq 60$ years group was $54.85 \%$ [95\%CI 49.91, 59.71], (Table 1).

Table 1: Two-by-two tables showing accuracy of the HHIE-S for identifying hearing loss in adults.

\begin{tabular}{|l|c|c||c|c|}
\hline & \multicolumn{2}{|c||}{$<60$ years of age } & \multicolumn{2}{c|}{$\geq 60$ years of age } \\
\hline Hearing loss criteria & HHIE $<\mathbf{8}(\%)$ & HHIE-S $\geq 8(\%)$ & HHIE <8 (\%) & HHIE-S $\geq 8(\%)$ \\
\hline Normal hearing & $336(36.9)$ & $341(37.5)$ & $186(16.8)$ & $162(14.6)$ \\
\hline Hearing loss $(>25 \mathrm{~dB})$ & $66(7.2)$ & $167(18.3)$ & $226(20.4)$ & $533(48.1)$ \\
\hline Totals & $402(44.2)$ & $508(55.8)$ & $412(37.2)$ & $695(62.8)$ \\
\hline
\end{tabular}

HHIE-S, Hearing Handicap Inventory for the Elderly - Screening. 
The prevalence of audiometric hearing loss in participants aged $\geq 60$ years was $69.12 \%$ (95\%CI 66.32, 71.79). Participants aged <60 years had a lower prevalence $(25.60 \%$ [95\%CI 22.82, 28.59]) (Figure 1). There was an increased risk of having a HHIE-S score $>8$ associated with participants from rural areas compared to urban areas (OR 1.32 [95\%CI 1.11, 1.59]; $\left.X^{2}(1)=9.51 ; p=0.002\right)$. Participants in rural populations also had an increased risk of mild hearing loss (OR 1.73 [95\%CI 1.45, 2.06]; $\left.\mathrm{X}^{2}(1)=38.13 ; \mathrm{p}<0.001\right)$ and disabling hearing loss (OR 2.04 [95\%CI 1.55, 2.67]; $\left.\mathrm{X}^{2}(1)=27.28 ; \mathrm{p}<0.001\right)$. There were no differences in hearing aid uptake (mild hearing loss: OR 0.93 [95\%CI 0.66, 1.46]; $X^{2}(1)=0.008 ; p=0.927$ ) (disabling hearing loss: OR 0.54 [95\%CI 0.84, 2.20]; $\left.\mathrm{X}^{2}(1)=1.614 ; \mathrm{p}=0.204\right)$ (Tables $2 \& 3$ ).

Table 2: A comparison of prevalence and degree of hearing loss (HL) and use of hearing aid(s) (HA) in urban and rural areas (all ages).

\begin{tabular}{|l|c|c||c|c|}
\hline & \multicolumn{2}{|c||}{ Urban (n = 982) } & \multicolumn{2}{c|}{ Rural (n = 1090) } \\
\hline Hearing loss criteria & HL (\%) & HL + HA (\%) & HL (\%) & HL + HA (\%) \\
\hline Mild HL & $409(41.6)$ & $49(5.0)$ & $602(55.2)$ & $53(4.9)$ \\
\hline Disabling HL & $88(9.0)$ & $29(2.9)$ & $182(16.7)$ & $43(3.9)$ \\
\hline Totals & $675(50.6)$ & $105(15.6)$ & $788(83.3)$ & $72(9.1)$ \\
\hline
\end{tabular}

Table 3: Analysis of participants with hearing loss (HL) and participants with hearing loss using hearing aids $(\mathrm{HL}+\mathrm{HA})$ in urban and rural areas (all ages).

\begin{tabular}{|l|c|c|c|c|}
\hline & \multicolumn{4}{|c|}{ Rural compared to urban locality } \\
\hline Hearing loss criteria & $\boldsymbol{X}^{2}(1)$ & $\boldsymbol{p}$ & OR & $\mathbf{9 5 \%}$ CI \\
\hline Mild HL & 38.13 & $<0.001$ & 1.73 & $1.45,2.06$ \\
\hline Disabling HL & 27.28 & $<0.001$ & 2.04 & $1.55,2.67$ \\
\hline Mild HL + HA & 0.008 & 0.927 & 0.93 & $0.66,1.46$ \\
\hline Disabling HL + HA & 1.614 & 0.204 & 0.54 & $0.84,2.20$ \\
\hline
\end{tabular}

CI, confidence interval; OR, odds ratio.

\section{DISCUSSION}

This study examined the prevalence of hearing loss in a cohort of self-referring adults from rural and urban areas of Western Australia. Participants in rural areas showed a significantly higher prevalence of hearing loss but their rates of hearing aid uptake were not significantly different. There was a high prevalence of hearing loss in those attending community-based 
hearing screenings and the HHIE-S questionnaire was a sensitive, but not specific, measure for detecting a hearing loss for adults aged $\geq 60$ years in this study. Approximately $70 \%$ of participants aged $\geq 60$ years had an audiometric hearing loss. However, these prevalence figures cannot be generalised to the wider population due to the sampling method. Population-based estimates of self-reported hearing loss are $46.2 \%$ for a regional population at 60-65 years of age. ${ }^{9}$ Whilst lower than our sample, this illustrates the high prevalence of self-reported hearing loss in the general population. Whilst this study did not examine the causes of hearing loss, both workers and families living in rural areas are at a higher risk of occupational noise exposure through agricultural and production industries. ${ }^{4}$

Our findings show an increased prevalence of hearing loss in regional and rural areas, and support previous calls ${ }^{9}$ for the better identification of hearing loss in primary care. Identifying and treating hearing loss in the pre-retirement period is associated with an increased uptake of hearing aids and improved rehabilitation outcomes. ${ }^{3,11}$ Considering the results of this study, the routine use of questionnaires for adults aged $\geq 60$ years in a primary care may enable the early identification of hearing loss, due to the high prevalence of the condition in this age group. However, self-report hearing measures do not have comparable accuracy to audiometry, and better access to these services in rural and remote areas is therefore still needed.

For both age groups the HHIE-S was sensitive $(\geq 60 \mathrm{yrs}=76.69 \% ;<60 \mathrm{yrs}=71.67 \%)$, but not specific $(\geq 60 \mathrm{yrs}=45.15 \% ;<60 \mathrm{yrs}=49.63 \%)$. Low specificity could result in excessive referrals for patients who self-report a hearing difficulty in the absence of an audiometric hearing loss. This study produced similar sensitivity estimates to previous studies of the HHIE-S (Sindhusake et al. 2001; 80\%) ${ }^{17}$, but with lower specificity (Sindhusake et al. 2001; $76 \%)$.

The HHIE-S false-positive rate for patients aged $<60$ years was substantially higher $(67.12 \%)$ compared to those aged $\geq 60$ years $(29.77 \%)$. False-positives incorrectly diagnose a patient as having a condition (i.e. self-reported hearing loss), in the absence of the condition according to the gold standard (i.e. hearing loss confirmed by audiometry). The impact of falsepositives in the $<60$ years group is likely to put greater strain on specialist services - resulting in an increase in referrals for hearing assessment in the absence of a hearing loss. However, it may be argued that a client who self-reports difficulty may still benefit from consultation. 
The $<60$ year-old group had a lower false-negative rate than the $\geq 60$ year old group, at $16.42 \%$ and $54.85 \%$, respectively. This is expected, considering the high prevalence of hearing loss in the $\geq 60$ years age group. These false-negatives represent patients with a hearing loss according to audiometric testing, who did not self-report a hearing loss using the HHIE-S. Whilst these patients may not feel they are experiencing sufficient limitations on their quality of life to warrant intervention, counselling on the impact of hearing loss and the benefits of early intervention may be beneficial. ${ }^{18}$

The cost-effectiveness of audiometric screening in community settings has been debated and there is some evidence that hearing screening increases the uptake of hearing aids. ${ }^{19}$ However, both screened and not-screened groups in the Yueh et al. (2010) ${ }^{19}$ study showed a low-uptake of hearing aids when followed-up one year after screening.

Pronk and colleagues ${ }^{20}$ have argued for better methods of hearing screening in adults and a wider range of alternative rehabilitative options in addition to hearing aids, such as informational counselling and communication strategies. In our study the HHIE-S showed poor specificity for identifying a clinically significant, audiometric hearing loss. This suggests that self-report measures may identify patients with listening difficulties, in the absence of audiometric hearing loss, who could benefit from these alternative rehabilitation options. This finding also highlights the need for better access to specialist hearing services in rural areas to distinguish between patients who would benefit from hearing aids, medical or surgical intervention or communication tactics.

Whilst screening questionnaires can be effective in detecting hearing loss early, these methods are not sufficient for a definitive diagnosis, which requires a diagnostic audiometry assessment. These services are rarely available in rural and remote areas ${ }^{21}$ and result in increased time and cost to travel to the nearest specialist, or require a specialist visit the region. The advent of automated audiometers ${ }^{22,23}$ that can be operated via telehealth or local primary care staff and interpreted remotely by audiologists may serve as a means of improving access to these specialist services. This technology, in conjunction with self-report measures, has the potential to streamline the clinical pathway of patients from identification to appropriate diagnosis and intervention. 


\section{LIMITATIONS}

As participants voluntarily presented for hearing screening it can be assumed that many already had concerns about their hearing and were therefore not representative of the general population. Poor specificity of the HHIE-S in this study is in contrast to other studies where specificity has ranged from $70-95 \% .{ }^{17,24}$ However, numerous population-based studies support the recommendation of regular, primary-care assessments for self-reported hearing loss, whether via a single-question or validated questionnaire..$^{7-9}$

\section{CONCLUSION}

The results highlight the increased risk of hearing loss in rural areas. This study demonstrates the potential value of self-report measures for detecting hearing impairment for adults, particularly those aged $\geq 60$ years in setting where audiometric screening equipment is not available. However, greater provision of audiometry services would allow more accurate identification of hearing loss.

\section{ACKNOWLEDGEMENTS}

We would like to acknowledge the Lions Hearing Foundations of Western Australia who provide free hearing screening across Western Australia and the volunteers who were involved in the collection of data used in this study. We would also like to particularly acknowledge the contribution of Ms Christine Smelt, Chairperson of the Lions Hearing Foundation of Western Australia and Ms Sharon Safstrom, Manager, The Avant CENTER, Ear Science Institute Australia. CGBJ received research funding from the Lions Hearing Foundation (WA).

\section{REFERENCES}

1. WHO. Millions living with hearing loss. Geneva: World Health Organisation, 2013.

2. Olusanya BO, Neumann KJ, Saunders JE. The global burden of disabling hearing impairment: a call to action. Bulletin of the World Health Organization 2014;92:367-73.

3. Dalton DS, Cruickshanks KJ, Klein BE, Klein R, Wiley TL, Nondahl DM. The impact of hearing loss on quality of life in older adults. The Gerontologist 2003; 43: 661-668.

4. Lower T, Fragar L, Depcynzksi J, Challinor K, Mills J, Williams W. Improving hearing health for farming families. Rural and Remote Health 2010; 10: 1350.

5. Smits C, Kapteyn TS, Houtgast T. Development and validation of an automatic speech-innoise screening test by telephone. International Journal Audiology 2004; 43: 15-28.

6. McShefferty D, Whitmer WM, Swan IR, Akeroyd MA. The effect of experience on the sensitivity and specificity of the whispered voice test: a diagnostic accuracy study. BMJ Open 2013; 3: e002394. 
7. Torre P, Moyer CJ, Haro NR. The accuracy of self-reported hearing loss in older LatinoAmerican adults. International Journal Audiology 2006; 45: 559-62.

8. Ramkissoon I, Cole M. Self-reported hearing difficulty versus audiometric screening in younger and older smokers and nonsmokers. Journal of Clinical Medicine Research 2011; 3: 183-190.

9. Swanepoel D, Eikelboom RH, Hunter ML, Friedland PL, Atlas MD. Self-reported hearing loss in baby boomers from the busselton healthy ageing study: audiometric correspondence and predictive value. Journal of the American Academy of Audiology 2013; 24: 514-521.

10. Laplante-Lévesque A, Hickson L, Worrall L. Rehabilitation of older adults with hearing impairment: a critical review. Journal of Aging and Health 2010; 22: 2.

11. Stephens SD, Meredith R, Callaghan DE, Hogan S, Rayment A. Early intervention and rehabilitation: factors influencing outcome. Acta oto-laryngologica Supplementum 1990; 476: 221225.

12. Newman CW, Weinstein BE, Jacobson GP, Hug GA. The Hearing Handicap Inventory for Adults: psychometric adequacy and audiometric correlates. Ear and Hearing 1990; 11: 430-433.

13. Lichtenstein MJ, Hazuda HP. Cross-cultural adaptation of the hearing handicap inventory for the Elderly-Screening Version (HHIE-S) for use with Spanish-speaking Mexican Americans. Journal of the American Geriatrics Society 1998; 46: 492-498.

14. Öberg M, Lunner T, Andersson G. Psychometric evaluation of hearing specific self-report measures and their associations with psychosocial and demographic variables. Audiological Medicine 2007; 5: 188-99.

15. Tomioka K, Ikeda H, Hanaie K, et al. The Hearing Handicap Inventory for Elderly-Screening (HHIE-S) versus a single question: reliability, validity, and relations with quality of life measures in the elderly community, Japan. Quality of Life Research 2013; 22: 1151-1159.

16. AIHW. Rural, regional and remote health: A guide to remoteness classifications. Canberra: Australian Institute of Health and Welfare, 2004.

17. Sindhusake D, Mitchell P, Smith W, et al. Validation of self-reported hearing loss. The Blue Mountains Hearing Study. International Journal of Epidemiology 2001; 30: 1371-1378.

18. Salonen J, Johansson R, Karjalainen S, Vahlberg T, Isoaho R. Relationship between selfreported hearing and measured hearing impairment in an elderly population in Finland. International Journal Audiology 2011; 50: 297-302.

19. Yueh B, Collins MP, Souza PE, Boyko EJ, Loovis CF, Heagerty PJ et al. Long-term effectiveness of screening for hearing loss: the Screening for Auditory Impairment - Which Hearing Assessment Test (SAI-WHAT) randomized trial. Journal of the American Geriatrics Society 2010; 58: 427-434.

20. Pronk M, Kramer SE, Davis AC, Stephens D, Smith PA, Thodi C, et al. Interventions following hearing screening in adults: A systematic descriptive review. International Journal of Audiology 2011; 50: 594-609.

21. AIHW. Medical labour force 2009. Canberra: Australian Institute of Health and Welfare, 2011.

22. Swanepoel D, Mngemane S, Molemong S, Mkwanazi H, Tutshini S. Hearing assessmentreliability, accuracy, and efficiency of automated audiometry. Telemedicine Journal and e-health 2010; 16: 557-563.

23. Eikelboom RH, Swanepoel D, Motakef S, Upson GS. Clinical validation of the AMTAS automated audiometer. Int J Audiol 2013;52:342-9.

24. Nondahl DM, Cruickshanks KJ, Wiley TL, Tweed TS, Klein R, Klein BEK. Accuracy of Self-reported Hearing Loss. International Journal of Audiology 1998;37:295-301. 